\title{
Transgranular Pore Formation in Nanocrystalline Coatings Condenced in the "Openess" of Vacuum and Quasi-Closed Volume
}

\author{
Leonid Skatkov", Petr Cheremskoy", Anatoliy Pugachev², Alexander Panikarskiy², \\ Valeriy Gomozov ${ }^{3}$ \\ ${ }^{1}$ Technical Division, Printed Circuit Board "Argo" Ltd., Beer Sheva, Israel; ${ }^{2}$ Physics of Metals and Semiconductors Department, \\ National Technical University "KhPI", Kharkov, Ukraine; ${ }^{3}$ Electrochemistry Department, National Technical University "KhPI", \\ Kharkov, Ukraine. \\ Email: " sf_lskatkov@bezeqint.net,tgg@kpi.kharkov.ua, zx47@list.ru
}

Received June $2^{\text {nd }}, 2013$; revised July $4^{\text {th }}, 2013$; accepted July $22^{\text {nd }}, 2013$

Copyright (C) 2013 Leonid Skatkov et al. This is an open access article distributed under the Creative Commons Attribution License, which permits unrestricted use, distribution, and reproduction in any medium, provided the original work is properly cited.

\begin{abstract}
It has presented a review of the results of the survey on the formation and behavior of submicropores (SMP) in monoand poly-crystalline systems, condensed in the "open vacuum" and quasi-closed volume. The mechanisms, patterns and characteristics of these processes, depending on the physical and technological factors are reviewed.
\end{abstract}

Keywords: Pore; SAXS; PbS; CdTe; Au; Vacuum

\section{Introduction}

It has presented a review of the results of the survey on the formation and behavior of submicropores (SMP) in mono- and poly-crystalline systems, condensed in the "open vacuum" and quasi-closed volume. The mechanisms, patterns and characteristics of these processes, depending on the physical and technological factors are reviewed. Development of new materials for different areas of technology is substantially related to a porosity problem, which considerably affects the properties of materials and process characteristics. Different crystalline and amorphous condensed systems, such as coatings, multilayer modulated structures are widely applied. Their typical structural components are pores of various dispersity, shape and nature of the location in the volume. The results of [1-4] indicate that pore formation in these systems is caused due to the laws of the formation of amorphous and crystalline structures at condensation of the substance. The main factors that determine the nature of the pore formation process at condensation are the followings: competition of diffusion and sorption processes, gas emission and adsorption, the orienting effect of the molecular flow, ratio of supercooling, evaporation and condensation degree, as well as the structural condi-

${ }^{*}$ This author deceased on 20 June 2005.

${ }^{\#}$ Corresponding author. tion, chemical composition and topology of the substrate surface and distinction of its thermal dilatation of condensate.

Mechanisms of pore formation at condensation-vacancy diffusion, sorption of gases, the "shadow" effect, loose fusion of elements of the structure and growth inhomogeneity - act together in different proportions depending on the physical conditions of formation process and structure of condensate, and are subject to simulation.

In order to conduct such simulation in practice, the issue of location of preferred formation of pores of different dispersion in conditions of domination of each mechanism is of utmost importance. Therefore, this paper discusses the results of survey on submicroporosity in mono- and poly-crystalline condensates for the purpose to identify the main factors, which cause or limit intragranular pore formation processes.

\section{Experimental}

Condensed surfaces of $\mathrm{Au}, \mathrm{PbS}, \mathrm{CdTe}$ and dispersion strengthening vacuum condensates $\mathrm{Cu}-\mathrm{Al}_{2} \mathrm{O}_{3}$ (concentration of $\mathrm{Al}_{2} \mathrm{O}_{3} \leq 0.1$ mass $\%$ ) have been inspected by the methods of X-ray structure analysis (XSA), small-angle $\mathrm{X}$-ray scattering (SAXS), transmission electron microscopy (TEM) as well as by precise measurement of hydrostatic density. The research methods are described in 
[2-4]. Influence of insoluble and soluble admixtures on the pore formation condensation processes illustrate research results of dispersion strengthening compositions (DSC) of copper, nickel and permalloy $\left\{\mathrm{Cu}-3 \% \mathrm{Al}_{2} \mathrm{O}_{3}\right.$, $\mathrm{Cu}-(0.5-3) \% \mathrm{SiO}, \mathrm{Ni}-(0.5-5.6) \% \mathrm{SiO}, 80 \% \mathrm{Ni}-$ $20 \% \mathrm{Fe}-(0.5-3.5) \% \mathrm{SiO}, 83 \% \mathrm{Ni}-17 \% \mathrm{Fe}-(1.5-$ $3) \% \mathrm{SiO}$ \} having a width of $5-20 \mathrm{mkm}$, condensed from steam-type phase on sitall substrates with a polycrystalline sublayer of $\mathrm{NaCl}$ at $\mathrm{T}=200^{\circ} \mathrm{C}-380^{\circ} \mathrm{C}$ in vacuum 2 $5 \times 10^{-3}$ Torr and simultaneous evaporation from various coolled molds, arranged so that the direction of incidence of molecular flow contemporaneously evaporated onto a substrate material, appeared to be close to normal. For evaporation of copper, nickel and permalloy it has been used crucible and electron beam methods, while maintaining condensation speed rate of $8-10 \mathrm{~nm} / \mathrm{sec}$. Evaporation of $\mathrm{Al}_{2} \mathrm{O}_{3}$ and $\mathrm{SiO}$ has been conducted by resistance method with condensation speed rate of $1-2 \mathrm{~nm} / \mathrm{sec}$ at separation of molecular flow to prevent carryout of oxide particles from evaporator on the substrate. Alloying of $83 \% \mathrm{Ni}-17 \%$ Fe permalloy condensates by $\mathrm{Si}(1 \%-3 \%)$ has been conducted by induction and separation evaporation of charge (permalloy + silicon) from one mold at condensation speed rate of $1.3-14 \mathrm{~nm} / \mathrm{sec}$.

\section{Research Results}

Results, achieved in this paper relate to formation of condensation pores mainly in the scope of the condensate structural elements. It has become possible to reveal the role of mechanisms which cause intragranular pore formation processes, particularly at the initial stages of condensate formation. It is mostly concern SMP, revealed by SAXS and TEM methods.

\subsection{Condensation in "Open" Vacuum}

Submicroporous structure of condensate can be revealed quite clearly, for instance, in thin $(\mathrm{h} \leq 100 \mathrm{~nm}) \mathrm{Au}$ films (see Figure 1 sation (molecular flow incidence angle $\beta=0)$ with quite high speed $\left(\omega_{k} \geq 10 \mathrm{~nm} / \mathrm{sec}\right)$, at room temperature in vacuum of $\sim 10^{-3} \mathrm{~Pa}$. The nature of the anisotropy SAXS in these films at different arrangement of samples as to initial X-ray beam (Figure 1) testifies that most of SMP are elongated and arranged along the normal as to the film.

An average value of the mean size of the SMP, calculated by Guinier method [5], in condensates with thickness of $125 \mathrm{~nm}$ are equal $6-10 \mathrm{~nm}$ in the area of film, 20 - $40 \mathrm{~nm}$ along the normal, while the total volume concentration of SMP is $1 \%-2 \%$. This corresponds to SMP size in the area of condensate, calculated based on the data of TEM films. Elongated shape of SMP arranged along the filmcontributes greatly to their electron and

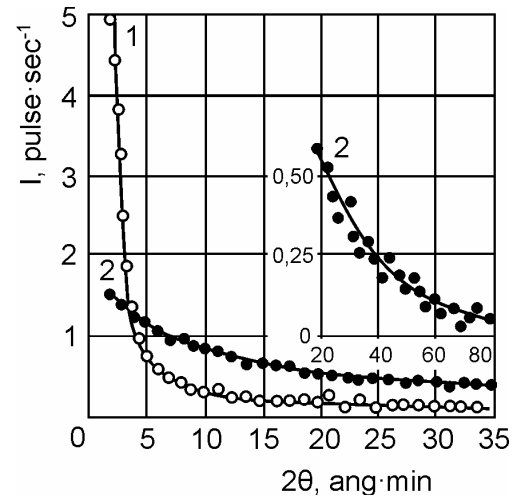

Figure 1. SAXS indicatrix for thin $(h=125 \mathrm{~nm})$ Au polycrystalline films: 1 , slope survey (under $45^{\circ}$ angle), 2 , normal survey.

microscopic identification.

Considering the abovementioned conditions of condensate formation it shall be pointed out that vacancy diffusion mechanism appears to be the predominant mechanism of intragranular pore formation in Au films. All other mechanisms resulting in formation of transgranular pores are of much smaller effect at the chosen conditions of condensation and normal incidence of the molecular flow. Amount transgranular poresis much less, while their volumetric concentration is much higher.

Intragranular pore formation is greatly improved by the processes of residual gases capture by the films. In $\mathrm{Au}$ condensates, being inspected, gas-filled submicropores forma kind of a grid inside the grains, cells of which have polyhedral shape, and slightly differs from each other the by the image contrast.

Along with formation and growth of intragranular and transgranular pores, at reduction of the number of intragranular stoppers due to secondary processes occurred in the fillm within post-condensation period, at sufficient thermal activation it might take place partial or complete dissolution of intragranular pores with their further carryout at the border between the structural elements. Similar processes have been observed in non-crucible normally condensed polycrystalline Ni films (Figure 2), where as a result of post-condensation annealing intragranular pores are formed on interblock boundaries. Enlargement of the structural elements due to recrystallization, accompanied by displaying of extinction contours of condensate images with transmission electron microscope, certifies the improvement of films, stimulated by heating. Mechanisms of pore formation condensation processes in monocrystalline films, condensed in "open" vacuum, can be detected by observing of condensation formation process of the same substance, but in different structural states, at changing of only $\mathrm{T}_{\mathrm{n}}$, while other factors ( $\mathrm{h}, \omega_{k}, \beta$ and $\mathrm{p}$ ) remain constant. It has been implemented on plumbum sulfide films of 3 microns 


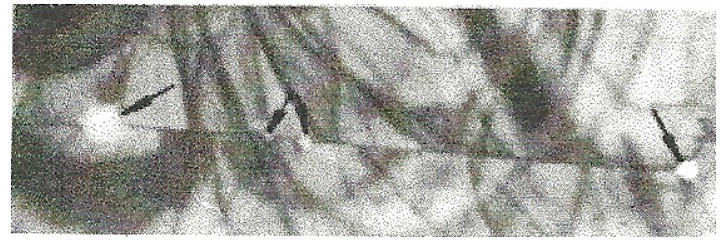

Figure 2. Pores formation due to secondary processes during isothermal $\left(850^{\circ}\right)$ vacuum heat of the uncrucible $\mathrm{Ni}$ films $\left(\mathrm{h} \approx 2 \mathrm{mkm}, \mathrm{T}_{\mathrm{II}}=110^{\circ} \mathrm{C}, \omega \approx \mathrm{nm} / \mathrm{sec}, \mathrm{p} \approx 10^{-3} \mathrm{~Pa}\right)$; $\times 10^{4}$.

thickness, condensed on chips $(100) \mathrm{NaCl}$ at $\omega_{k}=10$ $\mathrm{nm} / \mathrm{sec}, \quad \beta=0, \mathrm{p}=10^{-3} \mathrm{~Pa}$ in temperature range $30^{\circ} \mathrm{C} \leq T_{n} \leq 450^{\circ} \mathrm{C}$. It is observed a number of typical features, caused due to orienting effect of monocrystalline substrate, having a lattice similar to $\mathrm{PbS}$ and considerable difference of $\mathrm{NaCl}$ and $\mathrm{PbS}$ linear extension coefficients, on condensate. Film, prepared at $T_{n} \leq 80^{\circ} \mathrm{C}$, are appeared to be polycrystalline and have a dispersed structure with a strong crystallographic orientation (texture), the axis of which [100] coincides with the normal as to the film. With $\mathrm{T}_{\mathrm{n}}$ growth from $80^{\circ}$ to $100^{\circ} \mathrm{C}$ axial structure [100] maintain the same, and its perfection degree increases. At the same time, in the condensate from the side facing to the substrate, it is detected formation of $\mathrm{Pb}$ sulfide epitaxial monocrystalline layer, thickness of which increases with $\mathrm{T}_{\mathrm{n}}$ growth; at $T_{n} \geq 300^{\circ} \mathrm{C}$ the whole film appears to be a single crystal, epitaxially grown on $\mathrm{NaCl}$ chip [100]. Transition of textured polycrystal into a single crystal at the temperature range $250^{\circ} \mathrm{C} \leq T_{n} \leq 320^{\circ} \mathrm{C}$ is accompanied by the complete disappearance of the dark polycrystalline surface layer observed at lower $T_{n}$ values, and condensate surface acquires brilliance. It has earlier been shown that SAXS intensity, which considerably exceeds theoretically possible intensity of double Wulf-Bragg reflections within the entire $T_{n}$ range, which is caused due to porosity of the film. Spatial separation of SAXS extinguishes by clearly expressed anisotropy, the nature of which varies at $T_{n}$ changing (Figure 3).

At recording, corresponding to normal as to films surface (polar angle $\psi=0$ ) direction of X-ray beam and different azimuthal angles $\varphi$, in condensates received at $T_{n} \leq 80 \mathrm{C}$, it is observed isotropic SAXS with asymptotic of indicatrix proportional to $s$

$|s|=4 \pi \sin \Theta / \lambda \approx 4 \pi \theta / \lambda, 2 \Theta$-dispersion angle. Along with the axial texture [100] at $T_{n} \leq 80 \mathrm{C}$ on a small-angle X-ray picture it is revealed straight outlines of SAXS figure, caused by bounding of submicropores.At the same time asymptotic of indicatrix SAXS changes significantly as well. On small-angle X-ray pictures, which comply with recording at $\psi=0$ SAXS figure is displayed in a form of regular quadrilateral. At temperature growth up to $T_{n}=145 \mathrm{C}$ outlines of the figure become sharper and curvilinear, while multiple angledispersion range becomesslightly shorter. At inclined recording, which comply with $\psi=45$, SAXS figure is displayed in a form of elongated oval, getting straight outlines at $\mathrm{T}$ growth up to $145^{\circ} \mathrm{C}$ ( Figure 3(a)).

Revealed features of low-angle X-ray pictures are considered to be a reflection of regular arrangement of SMP having the shape of elongated parallelepipeds, the orientation of which remains constant up to $T_{n}=285^{\circ} \mathrm{C}$. Di-
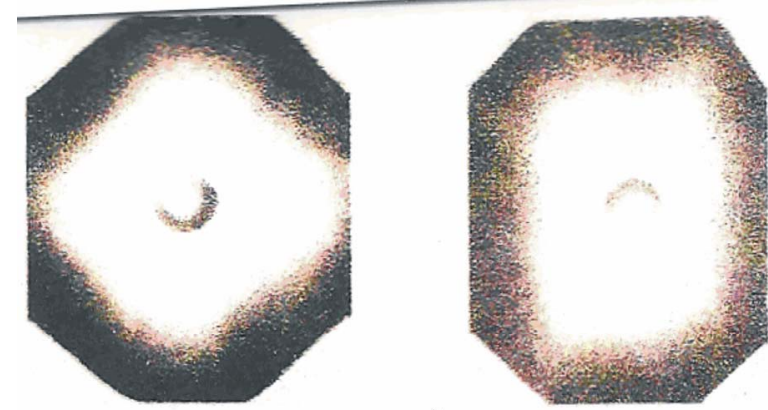

a)
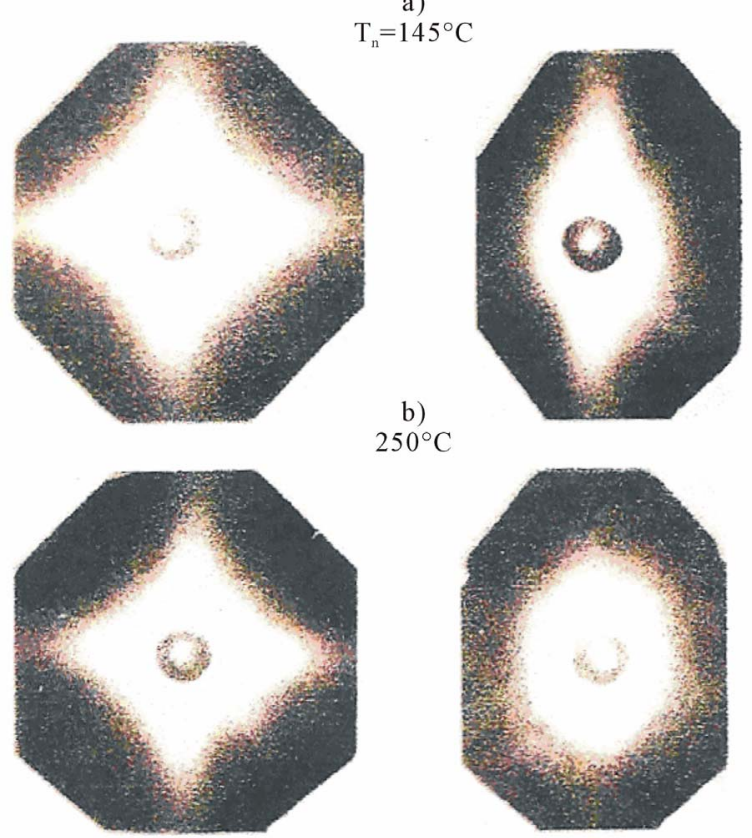

c)
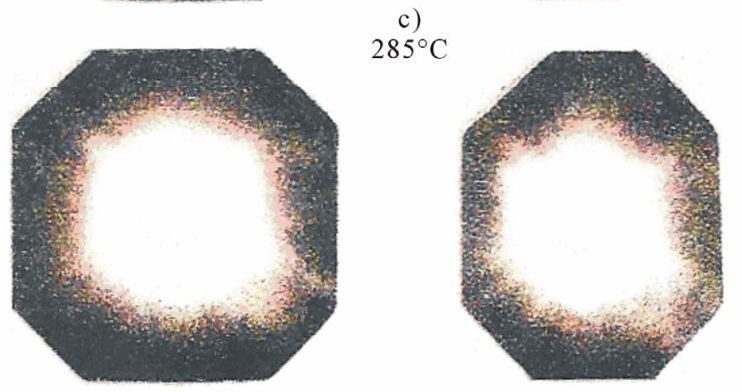

Figure 3. Small-angle X-ray picture of $\mathrm{PbS}$ epitaxial films condenced under the different $T_{n}$ : on the left-direct survey $\left(\Psi=0^{\circ}\right)$; on the left-inclined survey $\left(\Psi= \pm 45^{\circ}\right)$. 
agonal directions of the square on the angle X-ray $p$ tures coincide with crystallographic directions [001] and [010], while orientation of the small axis of rectangular (inclined recording), corresponds to projection of primary $\mathrm{X}$-ray beam direction at the direction of [100] monocrystalline epitaxial layer formed in condensates from the side facing the substrate. Growth of $T_{n}$ up to $250^{\circ} \mathrm{C}$ is accompanied by a significant change of SAXS image. At perpendicular $(\psi=0)$ recording it is observed degeneration of the square into cross-shaped figure with quite an uneven angular distribution of intensity (Figures 3(a) and (b)). Angular dispersion range increases to the same extent for the directions [001] and [010], but decreases for the direction [011]. Most of SAXS intensity is concentrated in the boundaries of the central part of small-angle $\mathrm{X}$-ray picture. Amplification of PMP anisotropy is also observed at inclined $(\psi=45)$ recording. At $\mathrm{T}_{\mathrm{n}}$ growth a rectangle revealed at $T_{n}=145^{\circ} \mathrm{C}$ rectangle is converted into a spindle-shaped figure which main axis is oriented along [100], and the angular range of dispersion along this axis occurs to be the same as for [001] and [010] at perpendicular $(\psi=0)$ recording. Along the minor axis SAXS range is much less than at lower values of $T_{n}$ (Figure 3(b)).

All this confirms that the processes of epitaxial layer buildup and axial texture [100] improvement in the studied condensates accompany by SMP anisometry increase, while SMP acquire correct shape caused due to mostly being bounded by plates of $\{100\}$ type. These SMP are oriented by the bounded plane along the growth of the epitaxial layer, which coincides with the direction of the predominant crystallographic orientation [100], i.e., along the direction of molecular flow incidence at condensation. The planes of $\{100\}$ type in PbS mono-crystal are considered to be cleavage planes and have a minimum surface energy equal to $\sim 0.6 \mathrm{~J} / \mathrm{m}^{2}$ for PbS. Thus, SMP bounding at epitaxial growth of condensate with predominantly such planes ("subtraction crystals") is completely natural process, as long as according to Gibbs-Curie-Wulff rule,for such crystals tract bounded with planes of $\{100\}$ type appears to be equilibrium. At $T_{n}=28^{\circ} \mathrm{C}$, when the most of condensate volume has already been occupied by an epitaxial monocrystal, $\mathrm{X}$-ray pictures, recorded at the angle $(\psi=45)$, display hexagon-shape figure with four angles of $135^{\circ}$ (Figure 3(c)). The central part of PMP to normally recorded $(\psi=0)$ X-ray pictures slightly expands along with the "tails" reduction in [001] and [010] directions. On inclined $(\psi=45)$ picture recorded in [001] direction, "tails" disappear almost entirely, and the main axis of the hexagon is equal to the side of the square, revealed in the central part of the normally recorded X-ray pictures. This means that SMP acquire an additional bounding $\{110\}$, and besides only those SMP are bounded, which scatter within the designated square, i.e. their larger fraction, and thus, SMP anisometry rate starts to decrease considerably. In the range of $285^{\circ} \mathrm{C} \leq T_{n} \leq 360^{\circ} \mathrm{C}$ PMP intensity is become dramatically weakened at changing of indicatrixes asymptotic, indicating a significant decrease in SMP concentration and anisotropy. At $T_{n}$ increase up to $380^{\circ} \mathrm{C}-400^{\circ} \mathrm{C}$ integrated intensity of SAXS increases as well, and SAXS indicatrixes recorded at $\psi=0$, get the asymptotic behavior of $\sim s^{-4}$ due formation of SMP with more uniform cross-section. The small-angle X-ray pictures recorded at $\psi=0$ show shortening of the dispersion angular range and decrease of dispersion anisotropy degree in all directions. Instead of a square shape PMP acquires a shape of relatively equiaxed hexagon (Figure 3(d)) as a result of additional bounding of SMP with $\{110\}$ planes which is particularly distinct at $T_{n} \geq 400^{\circ} \mathrm{C}$. Overall submicroporosity of epitaxial condensates, which equals to $1 \%$ at $T_{n}=145^{\circ} \mathrm{C}$, decreases from $0.6 \%$ at $\mathrm{T}_{\text {п }} \approx 200^{\circ} \mathrm{C}$ to $0.3 \%$ at $\mathrm{T}_{\text {п }}$, and at temperature increase up tо до $285 \mathrm{C}$ is further reduced by a half. This occurs at complete vanishing of polycrystalline textured fraction from condensate, when the whole film has already become an epitaxial monocrystal, which structure improvements accompanied by decrease in submicroporosity. So the lowest level of $\mathrm{C}_{\mathrm{M}} \approx 0.025 \%$ corresponds to the minimal ( $\sim 5$ ang.min) width of fluctuation curves at $T_{\Pi} \approx 365^{\circ} \mathrm{C}$. As to SMP dispersion, at increaseof epitaxial layer volume, it hardly changes and an average SMP size is $10-15 \mathrm{~nm}$. Polydispersity decreases simultaneously with the acquiring by the pores of clear bounding. Along with broadening of fluctuation curves and submicroporosity growth at $\mathrm{T}_{\Pi} \geq 370^{\circ} \mathrm{C}$ it is occurred SMP dispersion (Figure 3(b)) and heterogenity decrease at increase in their polydispersity. Thus, an interesting phenomenon is observed-fragmentation of "substracting" crystals (bounded SMP) as a result of plastic deformation of the epitaxial monocrystal at cooling from high $T_{п}$ under compressive thermal load arising in the film as a result of significant difference of linear expansion coefficients in $\mathrm{PbS}\left(1.9 \times 10^{-6}\right.$ degr. $\left.^{-1}\right)$ and $\mathrm{NaCl}\left(3.9 \times 10^{-6}\right.$ degr. $\left.^{-1}\right)$. This deformation is accompanied by an additional SMP bounding bysliding planes of $\{110\}$ type. As a result of this diffusion strands appearon SAXS figures along the direction [110] (Figure 3(d)). In monocrystal epitaxially grown films thermally activated SMP treatment occurs less rapidly than in polycrystal films of the same chemical composition and controlled by contentionof volume and surface diffusion mechanisms depending on the film thickness and extent of its structural perfection. The higher is the level of its thickness and perfection, the greater is the role volume diffusion in treatment process. Inspection of thermal effect on porosity and structure of such films has been executed by a vacuum $\left(\mathrm{p}=3 \times 10^{-3} \mathrm{~Pa}\right)$ annealing of the films non- 
separated from the condensate substrate of 3 microns thickness, condensed at a substrate temperature of $250^{\circ} \mathrm{C}$ and $400^{\circ} \mathrm{C}$, speed rate of $8 \mathrm{~nm} / \mathrm{s}$, and normal incidence of molecular flow. Heating of the films during $(9-54) \times 10^{2}$ sec has been performed directly after a cycle of condensation and cooling of the samples without depressurizing of the vacuum facility. To control initial structure of condensate samples from each film series has been left in non-annealing state and at heating of the remaining films have been located in vacuum facility at $\mathrm{T}=20^{\circ} \mathrm{C}$, and removed from it simultaneously with the other samples.

With heating temperature increase, angular scattering range shortens in all directions. Simultaneously, it is detected changing of the profile of small-angle X-rays pictures, recorded at different orientation of condensate as to the initial beam (Figure 4) and increase of SAXS indicatrix steepness. It is resulted due to SMP enlargement and shape changing during films annealing.

Reduction of the observed SAXS integrated intensity, indicates a decrease in overall submicroporosity. Based on the results of the pictures computer processing decrease in overall submicroporosity at annealing temperature increase is accompanied by a significant decrease in amount of small $(2 \mathrm{R} \leq 10 \mathrm{~nm})$ and an increase in amount of large $(2 R \geq 50 \mathrm{~nm}) \mathrm{SMP}$. In contrast to textured polycrystal condensates at annealing of monocrystal epitaxial films (in a selected temperature range) is has not observed any signs of cracks, which may occur during heating and cooling of "film-substrate" system as a consequence of the difference in thermal expansion coefficients of $\mathrm{PbS}$ and $\mathrm{NaCl}$. This is caused by more improved initial monocrystal structure of condensate capable to generate crack formation in films with less perfect structure. fies. Due to competition of kinetic and thermodynamic factors at pores growth or treatment it is revealed more or less strong tendency of their configuration changing. Figure 5 shows that at amplification of thermal activation it increases the intensity of transformation of small (less than $100 \mathrm{~nm}$ ) pores, which actually cause the recorded SAXS.

As a result of thermally activated structure improvement parallelepiped-shaped pores arranged in the direction of mono-crystal growth bounded with and basic planes, become more equiaxed at reduction of dislocation density and mosaic degree of crystal. At the same time, the larger the coherent scattering region (CSR)are and the more significant is a decrease in the dislocation density under the effect of heating, the more equiaxed shape the pores take and more delicate is a reduction of their volumetric concentration.

The following processes may cause changing of porosity characteristics, observed at heating of condensates:

1) "recondensation"-small pores "dissolve" at heating of condensates and settle on large ones, i.e. coalesce as a
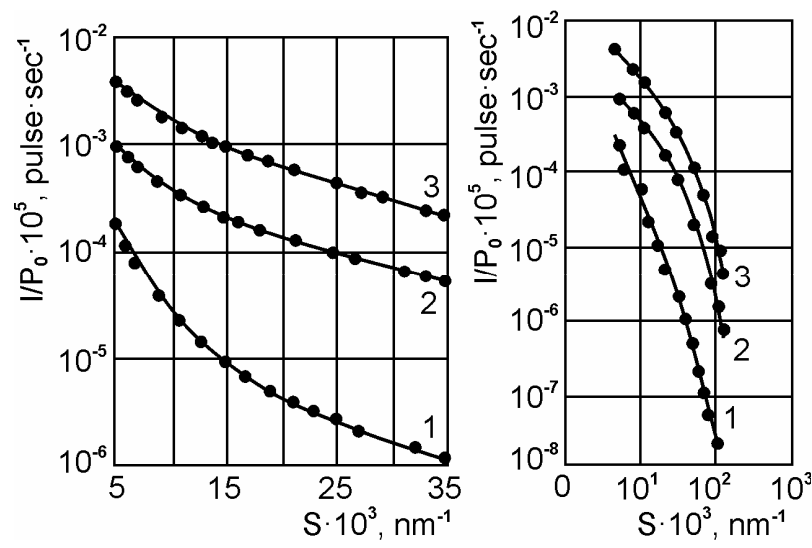

Figure 4. SAXS of the condenced Ni films: 1, Ni without impurities; $2, \mathrm{Ni}+1 \% \mathrm{SiO} ; 3, \mathrm{Ni}+5.6 \% \mathrm{SiO}\left(\mathrm{P}_{0}\right.$ : integral intensity of the X-ray primary beam).

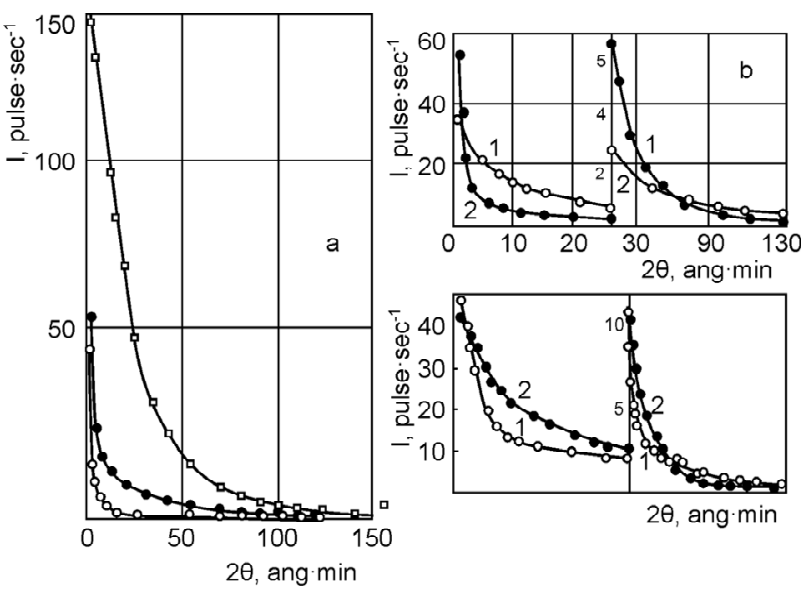

Figure 5. Impurities influence on the SAXS of permalloy condensed films: a) 1, Fe-Ni without impurities; 2, Fe-Ni + $0.5 \% \mathrm{SiO} ; 3, \mathrm{Fe}-\mathrm{Ni}+3.5 \% \mathrm{SiO}$; b) 1 , without impurities; 2 , $3 \% \mathrm{Si}, \mathrm{T}_{n}=250^{\circ} \mathrm{C} ;$ c) $1,3 \% \mathrm{Si}, \mathrm{T}_{n}=380^{\circ} \mathrm{C} ; 2$, after annealing.

result of self-diffusion, without changing the total porosity degree;

2) pore sintering as to mechanisms of volumetric and surface diffusion, causing decrease of total porosity;

3) annihilation of pores, located at intergranular (or interblock) borders, by their crawling along the border and emerge at the outer surface of the film as to the border diffusion mechanism, which is particularly facilitated by columnar type of structure elements of non-annealing condensates, while the overal porosity should decrease;

4) increase in SMP volume by means of volumetric self-diffusion due to excess vacancies remaining in nonannealing films, immured at condensation, or from complexes.

Depending on the particular porosity characteristics of the films in their initial state as well as conditions of their formation, these mechanisms may act in different com- 
binations. This intragranular pore formation corresponds to a), b) and d).

Increasing of condensation rate, for example, intensifies the processes of coalescence, sintering and increase of the pores growth rate at annealing due to formation of a large number of small thermally low-resistant SMP in the initial state. Activization of the coalescence processes in such pores causes reduction of SMP concentration and size at long-lasting storage of condensates Therefore, to obtain films with minimal porosity it is advised to increase the condensation speed rate at lower $\mathrm{T}$ and carry out the subsequent isothermal heating, rather than increasing the temperature and reducing condensation speed rate. This is explained due to the high thermal sensitivity of smaller SMP observed at low T and high condensation speed.

The question of the way how at pores presence, heating causes to intensification of both diffusion and chemical interaction in a solid matter appears to be of a particular interest. This can be demonstrated on the example of inspected $\mathrm{PbS}$ epitaxial films, heating of which causes oxidation of the surface layers. Additional sources and sinks for vacancies appeared in these condensates have chemical nature, while their activity is allocated at the free surface and inner surfaces of the section. Thermally activated oxidation is first of all a powerful source of excess vacancies for a grid, when oxidation kinetics is determined by the diffusion of metal ions in defect oxide grid. At the same time vacancy supersaturation is proportional to the ratio of self-diffusion and heterodiffusion coefficients. At heating, resulyed in oxidation of $\mathrm{PbS}$ monocrystal condensates, ambient oxygen acts as a pump, "pulling" of $\mathrm{PbS}$ monocrystal, $\mathrm{Pb}$ atoms (or ions $\mathrm{Pb}++$ ), which interact with the "counter" oxygen atoms (or ions $\mathrm{O}+$ ) and form an oxide layer over PbS film. At the same time, the excess vacancies formed in $\mathrm{Pb}$ grid units, accelerate "counter" diffusion process of $\mathrm{Pb} \rightarrow \mathrm{O}$. Specified "pump" model can be applicable for a large number of other film systems considering type of film interaction with the environment (or substrate).

$\mathrm{X}$-ray pictures of the inspected DSK reveals only reflections corresponding to matrix material. However, no changes in angular position of the diffraction peaks in the films, alloyed with dispersed $\mathrm{Al}_{2} \mathrm{O}_{3}$ and $\mathrm{SiO}$ admixtures at crucible evaporation of metal, have been revealed. Mean period of crystal grid of copper, nickel and permalloy films, reinforced with $\mathrm{Al}_{2} \mathrm{O}_{3}$ and $\mathrm{SiO}$, is hardly affected by an increase in the concentration of dispersed admixtures and is close to a table value for metals. Therefore we can conclude that at crucial evaporation specified admixtures do not dissolve in the crystal grid, but are distributed randomly over the volume of condensate in a form of local segregations, limiting migration mobility of condensed metal atoms, and dramatically impairing perfection degree of the gained condensate structure. What is typical, increase in concentration of insoluble admixtures leads to dispersion of the structure elements, but does not affect the degree of elastic microstrains.

In contrast, at silicon alloying it has been revealed an increase in period of matrix crystal grid, and integral width of diffraction peaks with increasing of admixtures concentration. This certifies that silicon is partially dissolved in matrix simultaneously contributing to dispersion of the condensate structure elements and the generation of internal structural microstrains, as well as partially forming local segregations. Particular reduction in period of crystal grid is observed at non-crucible alloying of permalloy with silicon monoxide at $\mathrm{SiO}$ concentration above $3.5 \%$, which to a certain extent is explained due to formation of weak solid solution of $\mathrm{SiO}$ in the matrix at the absence of crucible admixtures which inhibit dissolution of silicon monoxide in metal.SAXS in DSK and metal coatings without admixtures have a significant difference both in terms of the intensity rate and nature of its angular distribution. The intensity level of SAXS DSC exceeds this level in non-alloyed films by more than one level of the valueat significant broadening of anglular distribution range.

\subsection{Condensation in Quasi-Closed Volume}

Evaporation and condensation from molecular flow in the "open" vacuum does not always ensure the formation of monocrystal films, in particular those of AIIBVI type, due to considerable non-equilibriumity of film growth process when supersaturation of vapors on growing condensate border is significant and almost no close interaction between the vapor and condensed phases is observed. So condensation of CdTe in the "open" vacuum at relatively low $\mathrm{Tn}=20^{\circ} \mathrm{C}-380^{\circ} \mathrm{C}$ leads to low surface mobility of adsorbed atoms and molecules, resulting in substantial concentration of defects in epitaxial layers. Increase of $\mathrm{Tn}$ up to $400^{\circ} \mathrm{C}-450^{\circ} \mathrm{C}$ is accompanied by intensive re-evaporation of the substance from the gained condensate surface, which impedes obtaining of solid layers and leads to stoichiometry destruction of the films (depletion with light cadmium) due to different vapor pressures of the components and lack of volumetric interaction.

Under the quasi-closed volume it is usually implied a non-completely sealed area in which the change in steam density due to its outflow into the outer space within condensation process is negligible. This method allows to reduce the difference between temperatures of the evaporator and the substrate so that over-saturation at "steam-condensate" border has declined to the optimal 
value, and the condensation has been carried out in conditions close to the conditions of thermodynamic equilibrium.

The key feature of the quasi-closed volume method is that the vapor phase is concentrated in a small enclosed space bounded with hot walls, due to which steam molecules outflow from a thermodynamic process appears to be practically impossible and condensation is carried out under intensive exchange interaction of the growing layer with vapor phase. A significant advantage of AIIBVI films synthesis in the quasi-closed volume is high partial pressure of chalcogenide at concentration greater than the residual gas pressure in crucible by an amount close to 103.

Regardless of the gaseous phase composition, formation of epitaxial condensate on mica in the quasi-closed volume is carried out in two consecutive steps: a) formation of transition heteroepitaxial layer, b) film autoepitaxial growth with improvement of microstructure. It have been investigated monocrystal films CdTe with thickness of $6-10$ microns, condensed on mica chips in different residual gas atmosphere at a substrate temperature $\mathrm{T}_{\Pi}=490^{\circ} \mathrm{C}$ and the evaporator temperature $\mathrm{Tev}=$ $750^{\circ} \mathrm{C}$.

The investigated samples appears to be block monocrystals, having different rate of structural perfection with crystallographic orientation [111] along the normal to the outer surface. Depending on condensation intensity level of PMP in antireflection films CdTe in non-reflecting position is $1 \times 10-(4-5) \times 10^{-5}$ of the intensity value of initial X-ray beam. This is considerably less than in the abovementioned epitaxial PbS films, condensed from molecular flow in the "open" vacuum.

Condensation in the quasi-closed volume contributes to formation of monocrystals with a low content of local inhomogeneities of electron density. At the same time, hydrostatic density of CdTe films is substantially lower than the theoretical density of $7435 \times 103 \mathrm{~kg} / \mathrm{m}^{3}$ of massive monocrystals CdTe and varies in the range (7.15 $7.3) \times 103 \mathrm{~kg} / \mathrm{m}^{3}$, depending on the composition of residual gas atmosphere and $\mathrm{T} \Pi / \mathrm{Tev}$ ratio. This indicates that epitaxial films CdTe contain porosity of condensation origin, which to a great extent cause recorder

As compared to epitaxial films of PbS, SAXS indicatrixes differ substantially by significantly lower level and considerable steepness of intensity drop (Figure 6), indicating formation of considerably fewer in amount but sufficiently large in shape scattering inhomogeneities (mostly submicro - and micropores) in CdTe condensates. Thus condensation in the quasi-closed volume introduces peculiar features in the mechanisms condensation pore formation due to ensuring of more equilibrium thermodynamic conditions compared with condensation in the "open vacuum."
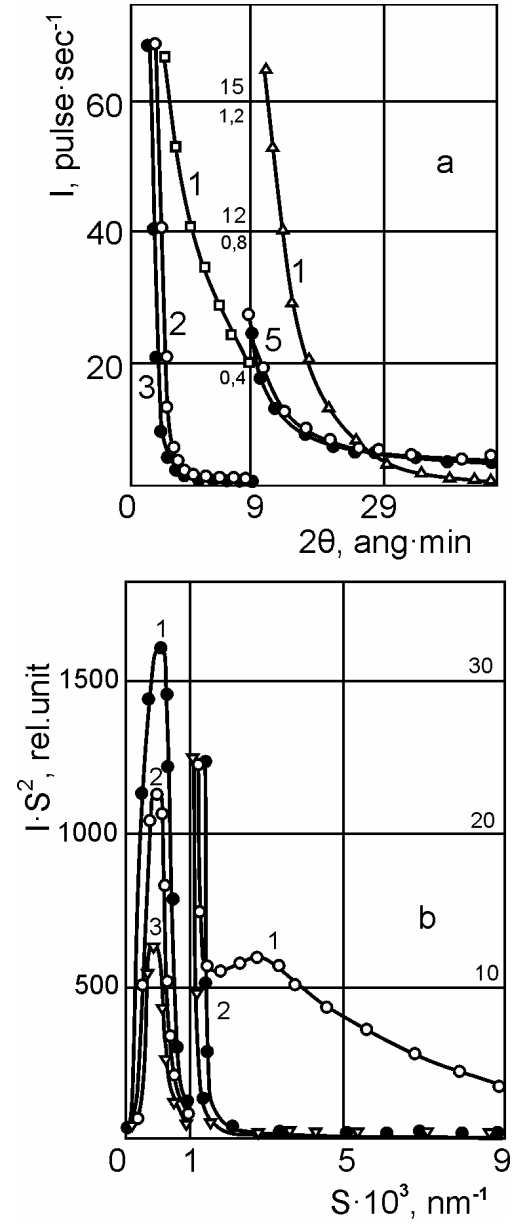

Figure 6. SAXS indicatrixes (a) and their invariants (b) of the epitaxial CdTe films condenced in quasi-closed volumes under the different temperatures $T_{n}$, vacuum $p=10^{-3}$ Pa: 1 , $\mathrm{T}=240^{\circ} \mathrm{C} ; 2, \mathrm{~T}=400^{\circ} \mathrm{C} ; 3, \mathrm{~T}=500^{\circ} \mathrm{C}$.

Conducting a comparison of SAXS films condensed residual gas atmosphere of various composition (Figure 7) it can be concluded volumetric concentration of SMP is extremely sensitive to composition of the gas environment at condensation. Most of SMP amount is formed at condensation in vacuum of $10-2 \mathrm{~Pa}$ with a predominant content of $\mathrm{O}_{2}$ (Table 1).

Features of films submicroporosity characteristics respond o their electrophysical characteristics. In particular, the most porous films are also featured with the highest carrier concentration $\left(1.44 \times 109 \mathrm{~cm}^{-3}\right)$ and traps for them.

At the same time, they contain peak concentration of small $(\mathrm{R} \leq 15 \mathrm{~nm}) \mathrm{SMP}$. The lowest amount $(0.8 \times 10 \%$ $2 \%$ vol.) of SMP is formed at condensation in the atmosphere of $\mathrm{H} 2$ at residual gas pressure rate of $10-2 \mathrm{~Pa}$. This is significantly lower than in films obtained in conventional vacuum atmosphere (Table 1) and is caused not only due to a significantly lower tendency of films to 


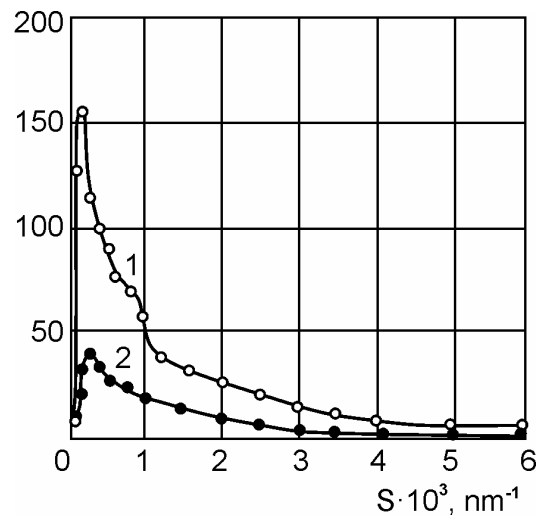

Figure 7. SAXS indicatrix invariant for CdTe epitaxial films condenced at difference temperatures of evaporator $T_{e v}$ and substrate $T_{n}$, vacuum $10^{-3}$ Pa: $1, T_{e v}=620^{\circ} \mathrm{C}, T_{n}=$ $300^{\circ} \mathrm{C} ; 2, \mathrm{~T}_{\mathrm{ev}}=710^{\circ} \mathrm{C} ; \mathrm{T}_{\mathrm{n}}=5_{00}^{\circ} \mathrm{C}$.

Table 1. Preparation conditions and characteristics of the epitaxial CdTe films $\left(\sigma-\right.$ specific conductivity $\times 10^{-9} \mathbf{O m}^{-1}$ $\mathrm{cm}^{-1} ; \mathbf{n}_{0}$-carriers concentration $\times 10^{8} \mathrm{~cm}^{-3} ; \mathrm{N}_{\mathrm{t}}$-traps concentration $\times 10^{16} \mathrm{~cm}^{-3} ; C_{M}$ and $2 R_{c}-$ volume concerntration and mean-weighted mean-square size of SMP).

\begin{tabular}{cccccc}
\hline $\begin{array}{r}\text { Working } \\
\text { atm., Pa }\end{array}$ & $\sigma$ & $\mathrm{n}_{\mathrm{o}}$ & $\mathrm{N}_{\mathrm{t}}$ & $\mathrm{C}_{\mathrm{M}}, \%$ & $2 \mathrm{R}_{\mathrm{c}}, \mathrm{nm}$ \\
\hline $10^{-3}$, vac. & 1.40 & 2.9 & 3.50 & 0.018 & 36 \\
$10^{-2}, \mathrm{H}_{2}$ & 0.05 & 0.1 & 0.02 & 0.008 & 50 \\
$10^{-2}, \mathrm{Ar}$ & 0.27 & 0.6 & 0.29 & 0.032 & 32 \\
$10^{-2}, \mathrm{O}_{2}$ & 7.00 & 14.4 & $10^{4}$ & 0.077 & 20 \\
\hline
\end{tabular}

internal oxidation during their formation in a $\mathrm{H}_{2}$ atmosphere in comparison with an atmosphere containing $\mathrm{O}_{2}$. Since atomic and ionic radii of hydrogen are significantly less than that of oxygen, its improved migratory ability facilitates CdTe diffusion in crystal grid without any delaysat sinks, sufficient for SMP formation, and also increases the possibility of $\mathrm{H}_{2}$ solutions formation in the matrix. Furthermore, formation of chemical compounds with hydrogen at a temperature close to $500^{\circ} \mathrm{C}$ is also not excluded.Condensates with a minimum porosity, obtained in a hydrogen atmosphere, also contain the lowest $\left(1 \times 10^{-7} \mathrm{~cm}^{-3}\right)$ carrier concentration and traps for them (Table 1). Films condesed in argon atmosphere, occupy an intermediate position between the films condesed in atmospheres of oxygen and hydrogen, as to their submicroporosity characteristics. Electrophysical properties of these films also correlate with the calculation data of SMP volumetric concentration (Table 1).Increasing of Тп from 240 to $500^{\circ} \mathrm{C}$ is accompanied by a decrease of the scattering ability of epitaxial films, indicating a significant decrease in the volumetric concentration of SMP, revealed by PMP method. This is illustrated with decrease of the area under the curves of PMP indicatrix invariants (Figures 8 and 9). Along with formation larger SMP at increased $\mathrm{T}_{\Pi}$ it is observed change in their size
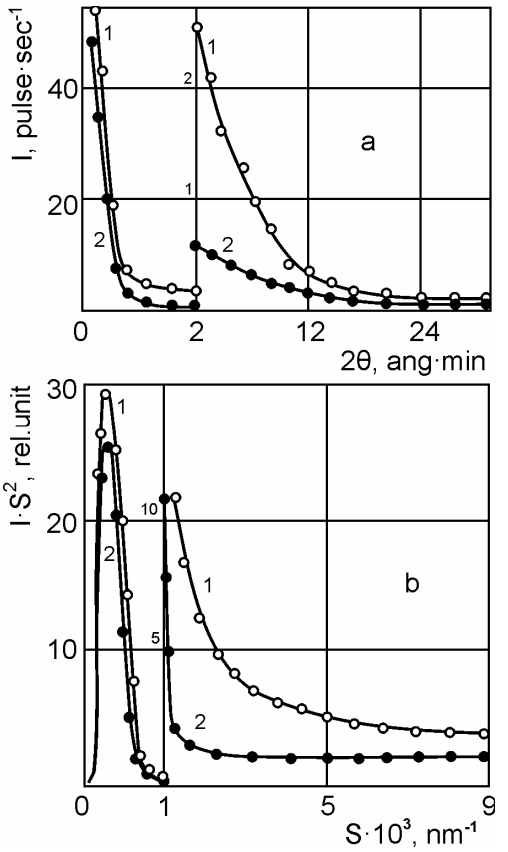

Figure 8. SAXS indicatrixes (a) and their invariants (b) for epitaxial CdTe films condenced at $\mathrm{Tn}-300^{\circ} \mathrm{C}$; $\mathrm{Tev}=670^{\circ} \mathrm{C}$, vacuum 10-3 Pa: 1 , stoichiometric composition batch; 2 , the surplus of Te.

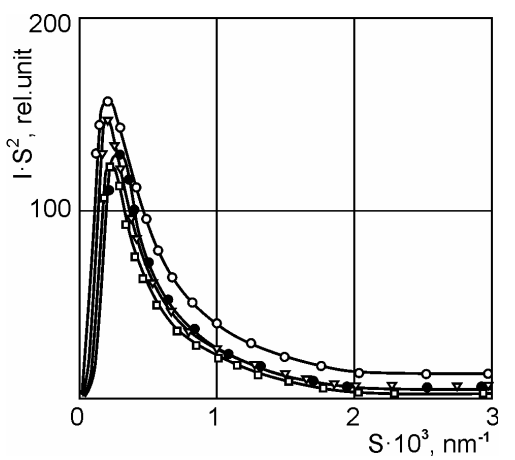

Figure 9. SAXS invariant indicatrix for CdTe epitaxial films condenced at $\mathrm{Tn}=490^{\circ} \mathrm{C}$ and same evaporation evaporation temperature $\left(750^{\circ} \mathrm{C}\right)$ at the difference gaseous atmosphere: $\circ-\mathrm{O}_{2}, \mathrm{p}=10-2 \mathrm{~Pa} ; \bullet-\mathrm{H}_{2}, \mathrm{p}=10$ - $2 \mathrm{~Pa}$; $\nabla-\mathrm{Ar}, \mathrm{p}=10-2$ Pa.

distribution, which is becomes more homogeneous. Tendency of SMP concentration reduction is revealed not only for thin, but also for thicker films. At the same time, thicker films (more than 20 microns thick) contain fewer SMP as compared to thicker concentrates [3,4].

In low-temperature films it is revealed obvious PMP anisotropy that is weakened with Tnincrease and atTп = $200^{\circ} \mathrm{C}$ is not observed [3]. Low angle records at different orientations of the samplesas to X-ray beam have allowed to reveal that SMP in low-temperature condensates CdTe are directed along the normal to the substrate. As it has been shown such shape and orientation are in- 
herent to the filmscondensed in the "open" vacuum.

Introduction of excess Te to the batch reduces the scattering ability of the films compared to CdTe films, condensed at the same Тп from the batch of undisturbed stoichiometric composition. At overal reduction of SMP volumetric concentration, volumetric contribution of small SMP increases significantly $(\mathrm{R} \leq 15-20 \mathrm{~nm})$ while concentration of large SMP with the size of more than 30 $\mathrm{nm}$ [3] decreases. This can be explained by the fact that the films condensed from the batch of stoichiometric composition appear to be depleted by more light $\mathrm{Cd}$ component as a result of its partial re-evaporation within condensate formation process. As it is known, during distillation of light components it is formed favorable conditions for diffusion pore formation by means of coalescence of vacancy complexes, as well as small SMP, which is confirmed experimentally, in particular, in the investigated coatings.

Therefore, excess of Te, specially introduced into the batch to achieve the stoichiometric composition formed by condensate, is an obstacle for pore formation processes of in epitaxial film.

As it os observed, the more equilibrium conditions are provided atmonocrystal condensate formation, the less active are the processes of condensation pore formation. It has been established experimentally that the most optimal conditions for formation of structurally perfect monocrystal films CdTe correspond to the relation of temperature ranges $\mathrm{T}=520^{\circ} \mathrm{C}-540^{\circ} \mathrm{C}$ and $\mathrm{T}=550^{\circ} \mathrm{C}$ $580^{\circ} \mathrm{C}$. It also relates to porosity of the inspected films, the level of which can be programmed according to the desired electrophysical characteristics of the films used in particular as base layers of thin-film photoelectric solar energy converters with optimal width of prohibited zone.

\section{Conclusions}

1) Intragranular condensation pore formation in nanocrystal systems is carried out by the joint action of vacancy diffusion and sorption mechanisms, depending on the intensity of gas emission or gas adsorption processes during condensation.

2) In conditions of condensates epitaxial growth, generated SMP formed acquires bounding made of crystallographic planes with minimal surface energy, in particular cleavage planes, forming the so-called "crystal subtraction", which inherits crystallographic orientation of the crystal-substrate.

3) Under compressive microstrain, arising during cooling of epitaxial condensates due to the difference of linear expansion coefficients of condensate and substrate, it is occurred deformation and grinding of "crystal subtraction" (bounded pores) by means oftheir additional bounding by sliding planes. Improvement of epitaxial coatings substructure during their post-condensation heating is accompanied by thermally activated processes of "crystal subtraction" coalescence and acquisition of equilibrium shape by them in accordance with thermodynamic criteria.

4) Introduction of foreign non-soluble admixtures to condensate contributes to development of intragranular porosity and stabilization of the porous structure. Partially soluble admixtures contribute to enlargement of condensation SMP.

5) Porosity of epitaxial coatings is most effectively minimized by means of condensation in quasi-closed volume under conditions of intensive exchange interaction of the growing layer with the vapor phase at optimal ratio between evaporation and condensation temperatures, which ensures an approximation to thermodynamic equilibrium conditions. Predominance of gas with high migratory ability, in particular, hydrogen, in the working atmosphere helps to minimize porosity of the film, which increases at the presence of inert gases and reaches a maximum level at condensation in the oxidizing atmosphere.

6) At isothermal heating of crystal coatings, it is occurred the processes of coalescence of excess vacancies and SMP of condensing interaction with formation of macropores which are partially "sintered" at long-lasting temperature exposure especially during recrystallization. In epitaxial condensates thermally activated treatment of SMP occurs less vigorously than in the polycrystal films of the same chemical composition and is controlled by competition of volumetric and surface diffusion mechanisms, depending on thickness andstructural perfection degree of the condensate. Heating of coating, non-separated from substrates causes formation of microcracks nuclei along the cleavage (sliding) plane sunder conditions of thermal stress arising due to different thermal dilatation of the film and the substrate.

7) Stabilizing effect of soluble admixtures on porosity is revealed in increase of the temperature at the start of SMP coalescence and sintering processes.

\section{REFERENCES}

[1] P. Cheremskoy, V. Slezov and V. Betechtin, "Pores in Solid State," Energoatomizdat, Moscow, p. 376.

[2] P. Cheremskoy, "Methods of Investigation of Solid State Porosity,” Energoatomizdat, USSR, 1985, p. 110.

[3] P. Cheremskoy, "Pores in Condensated in Compositioned System," Tes.Dr.Sci, Kharkiv, 2002, p. 32.

[4] A. Pugachev, A. Arinkin, N. P. Churakova, et al., "Lattice Dynamics and Substructure of Thin Aurumum Condensate Films," Physics of Metals and Metallography, Vol. 53, No. 5, 1982, pp. 911-916.

[5] O. Glater and O. Kratky, "Small Angle X-Ray Scattering," Academic Press, London, 1982. 